Article

\title{
Oral Microbiome in Four Female Centenarians
}

\author{
Yoshiaki Nomura ${ }^{1, *} \mathbb{0}$, Erika Kakuta ${ }^{2}$, Ayako Okada ${ }^{1}$, Ryoko Otsuka ${ }^{1}$, Mieko Shimada ${ }^{3}$, \\ Yasuko Tomizawa ${ }^{4}$, Chieko Taguchi ${ }^{5}$, Kazumune Arikawa ${ }^{5}$, Hideki Daikoku ${ }^{6}$, \\ Tamotsu Sato ${ }^{6}$ and Nobuhiro Hanada ${ }^{1}$ \\ 1 Department of Translational Research, Tsurumi University School of Dental Medicine, Kanagawa 230-8501, \\ Japan; okada-a@tsurumi-u.ac.jp (A.O.); otsuka-ryoko@tsurumi-u.ac.jp (R.O.); \\ hanada-n@tsurumi-u.ac.jp (N.H.) \\ 2 Department of Oral bacteriology, Tsurumi University School of Dental Medicine, Kanagawa 230-8501, Japan; \\ kakuta-erika@tsurumi-u.ac.jp \\ 3 Chiba Prefecture University of Health Sciences, Chiba 261-0014, Japan; mieko.shimada@cpuhs.ac.jp \\ 4 Department of Cardiovascular Surgery, Tokyo Women's Medical University, Tokyo 162-8666, Japan; \\ tomizawa.yasuko@twmu.ac.jp \\ 5 Department of Preventive and Public Oral Health, Nihon University School of Dentistry at Matsudo, \\ Matsudo 271-8587, Japan; taguchi.chieko@nihon-u.ac.jp (C.T.); arikawa.kazumune@nihon-u.ac.jp (K.A.) \\ 6 Iwate Dental Association, Morioka 470-2101, Japan; dai-koku@nifty.com (H.D.); \\ tamosato-dent@k-2inc.jp (T.S.) \\ * Correspondence: nomura-y@tsurumi-u.ac.jp; Tel.: +81-45-580-8462
}

Received: 29 June 2020; Accepted: 29 July 2020; Published: 31 July 2020

\begin{abstract}
The oral microbiome of healthy older adults has valuable information about a healthy microbiome. In this study, we collected and analyzed the oral microbiome of denture plaque and tongue coating samples from four female centenarians. After DNA extraction and purification, pyrosequencing of the V3-V4 hypervariable regions of the $16 \mathrm{~S}$ rRNA was carried out. The bacterial taxonomy for each lead was assigned based on a search of the EzBioCloud 16S database. We obtained a total of 199,723 valid, quality-controlled reads for denture plaque and 210,750 reads for tongue coating. The reads were assigned 407 operational taxonomic units with a $97 \%$ identity cutoff. Twenty-nine species were detected in both denture plaque and tongue coatings from all subjects. Firmicutes was the most abundant phylum; the Streptococcus salivarius group was the most abundant species in both the denture plaque and tongue coatings; and the Fusobacterium nucleatum group was detected in all subjects. In the bacterial profile, species formed clusters composed of bacteria with a wide range of prevalence and abundance, not dependent on phyla; each cluster may have specific species that could be candidates for a core microbiome. Firmicutes and Veillonella were abundant phyla on both plaque and tongue coatings of centenarians.
\end{abstract}

Keywords: oral microbiome; core microbiome; centenarian; next-generation sequencing

\section{Introduction}

The human microbiome changes with age [1] and health affects the composition of the microflora. It has been suggested that aging is accompanied by an underlying inflammatory state [2] that interacts with the microbiota of older adults and makes them more susceptible to age-related diseases [3-5]. Changes in the gut microbiome are explained by diseases, including metabolic changes and inflammatory conditions. Studies of intestinal microbiota in the elderly show that the microbiome affects a variety of clinical problems, including physical weakness, Clostridium difficile infection, colitis, vulvar vaginal atrophy, colorectal cancer, and atherosclerosis [6]. Other research has focused on the metabolism of nutrients. The microbiota has been shown to correlate with the declining metabolism of essential amino acids by aging [7]. 
In addition to the intestinal microbiome, the oral microbiome has been intensively studied. Both are affected by development, aging, and the state of oral disease. The formation of the first oral flora is strongly influenced by the mother [8-10]. Breast milk provides a source of bacteria that act as an inoculum for newborns [11-14]. The production and excretion of metabolites by pioneering colonies, such as Streptococcus and Actinomyces, alter the anaerobic oral environment. Under such conditions, anaerobic bacteria such as Veillonella and fusobacteria colonize [8,15]. With development, the microbial community evolves and microbial diversity increases $[16,17]$. Current knowledge shows that adult-like stability is reached around age two [15]. The established oral microbiota is disturbed by oral diseases. The development of dental caries has been associated with changes in microbial composition over time [18]. Differences in bacterial communities on the phylogenetic level were observed between healthy people and patients with periodontal disease $[19,20]$. The oral microbiota includes some pathogenic bacteria for systemic diseases. Therefore, the oral microbiome affects health and varies with health or disease [21]. Oral diseases, especially periodontal disease, can be the main risk factor for several systemic diseases [22-27]. Periodontal bacteria and their surface lipopolysaccharides were the agents of systemic diseases [28]. Recent advances of research on systemic diseases and oral health showed that there exist protective host factors for systemic diseases in relation to periodontal diseases [29-31]. The number of studies on the oral microbiota of community-dwelling older persons is limited [32-37].

In this study, denture plaque and tongue surface samples were obtained from people over 100 years old. The samples were analyzed by high-throughput sequencing of $16 \mathrm{~S}$ rRNA through a metagenomics approach. We describe the commonly prevalent and highly abundant species of four female centenarians and compare them with the healthy oral microbiome proposed in previous studies. In addition, differences in sampling sites and the co-prevalence of species were analyzed.

\section{Materials and Methods}

\subsection{Subject and Setting}

Originally, the aim of the survey was to investigate the relationship between oral health and systemic health in 80-year-old adults. This survey is known as the 8020 Data Bank Survey. A 20-year follow-up study was conducted from 1996 to 2017 with subjects 80 years of age (born in 1917) residing in the 8 districts served by one health center in Iwate Prefecture. The sampling method was cluster sampling, and the sampling frame was a complete count survey for all subjects. For the baseline survey, based on residential registration, public health nurses visited 944 homes where 80-year-olds lived, and 666 subjects participated in these checkups.

The follow-up survey was conducted by the resident register with surviving subjects who participated in the baseline survey. After 20 years, 12 subjects survived. Among them, 5 lived in their own home with their families; the others lived in nursing homes. Four subjects who lived in their own home agreed to participate in the survey. We visited their homes at 10:00 in the morning to minimize the effect of food intake. Details of the survey were described in our previous report $[27,28,37,38]$.

\subsection{Oral Examination}

Two dentists visited the homes of the subjects. An oral examination was carried out with a penlight and a dental mirror. The definition and diagnosis of dental caries were based on the criteria of the World Health Organization [39].

\subsection{Sample Collection}

As all subjects wore complete dentures, plaque samples were collected from the denture surface based on a method described in previous reports [40,41]. Briefly, the denture was brushed around the buccal surface with a toothbrush for $2 \mathrm{~min}$, followed by immersion of the toothbrush with the attached plaque in sterilized, phosphate-buffered saline (PBS). Tongue coating samples were collected by using 
a mucosal brush (ERAC 510Lion, Tokyo, Japan). The tongue surface was brushed one way, from back to front, 5 times. Samples were treated in the same way as denture plaque samples. Samples were kept on ice before being transported to the Iwate Dental Association in an ice box with refrigerant, then were stored at $-20^{\circ} \mathrm{C}$ for further analysis.

\subsection{Microbial DNA Extraction}

Denture plaque and tongue surface samples suspended in PBS were collected by a centrifuge at $3000 \mathrm{rpm}$ for $10 \mathrm{~min}$. DNA extraction was performed by a Maxwell 16 LEV Blood DNA Kit (Promega KK, Tokyo, Japan)according to the manufacturer's instructions. DNA concentration was measured by a NanoDrop ND-2000 (Thermo Fisher Scientific KK, Tokyo, Japan). Degradation of DNA was visually checked by electrophoresis on $1 \%$ agarose gel. Degradation of DNA and contamination of RNA were checked by a Qubit dsDNA BR Assay Kit (Thermo Fisher Scientific KK, Tokyo, Japan).

Samples meeting the following criteria were used for further sequence analysis: conc $>20 \mathrm{ng} / \mu \mathrm{L}$, volume $\geq 20 \mu \mathrm{L}, \mathrm{A} 260 / 280 \geq 1.8$, and A260/230 > 1.5. In this study, all samples met the criteria.

\subsection{Microbial Community Analysis}

Extracted DNA was analyzed in a laboratory (Chun Lab, Seoul, Korea). Polymerase chain reaction (PCR) amplification was performed using primers specific to the V3-V4 region pyrosequencing tags of the $16 \mathrm{~S}$ rRNA gene in the extracted bacterial DNA. Taxonomic classification of each read was assigned based on a search of the EzBioCloud 16S database [42,43], which contains the 16S rRNA genes of type strains that have valid published names and representative species-level phylotypes of both cultured and uncultured entries in the GenBank database, with complete hierarchical taxonomic classification from the phylum to species level [44].

\subsection{Bioinformatics Analysis}

The number of $16 \mathrm{~S}$ rRNA gene copies (absolute abundance) of operational taxonomic units (OTUs) was calculated by multiplying their respective relative abundance by the total number of $16 \mathrm{~S}$ rRNA gene copies. Bioinformatics analysis was performed by the microbiome package on the Bioconductor of R software [45].

\section{Results}

\subsection{Characteristics of Subjects Who Participated in this Study}

All four subjects who participated in this study were women, 100 years old, and wore complete dentures. One subject had five residual roots (sample ID 1), with complete dentures designed over the stump root. Blood tests and medical examinations were conducted to confirm the health of the subjects. Blood tests showed values in the normal range. Two subjects had hypertension. According to this continuous study, one subject had high blood pressure at age 85 (sample ID 1) and one had normal blood pressure at age 90 (sample ID 2). Other than that, there were no specific symptoms on examination.

\subsection{Sequence Data Details}

Out of eight samples from the four subjects, 199,723 reads for denture plaque (min, max: 42,792, $54,898)$ and 210,750 for tongue coatings $(45,193,57,753)$ passed quality control. From these reads, sequences clustered into 15 phyla, 31 classes, 49 orders, 71 families, 142 genera, and 406 species. A heatmap of all 407 species detected in this study is shown in Figure S1. All sequence data are provided in the Supplementary Materials.

The ACE, Chao1, jackknife, Shannon, and Simpson alpha diversity indices were calculated to analyze the diversity and richness of all samples. By comparing the samples of denture plaque and tongues, the ACE, Chao1, jackknife, and Shannon indices were not significantly different $(P>0.05)$, 
proving that bacterial diversity and richness were similar in the samples collected from denture plaque and tongues. The values of these indices are shown in Table S1. Statistics of taxonomic assignment are shown in Table S2. A rarefaction curve is shown in Figure S2.

\subsection{Oral Microbiome Profile of Centenarians}

Figure $1 \mathrm{~A}$ shows the relative abundance of detected bacteria at the phylum level. On both denture plaque and the tongue, Firmicutes were abundant. Figure 1B shows the phylum level proportion of bacteria of the four subjects. For three subjects, Firmicutes were abundant in both saliva and the tongue. Proteobacteria were relatively abundant in the saliva and tongue of one subject. Dynamic pie charts are presented in the Supplementary Materials.

(A)

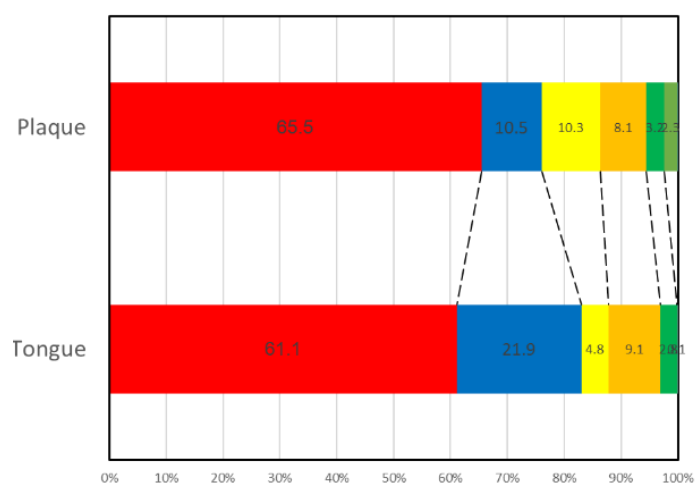

(B)

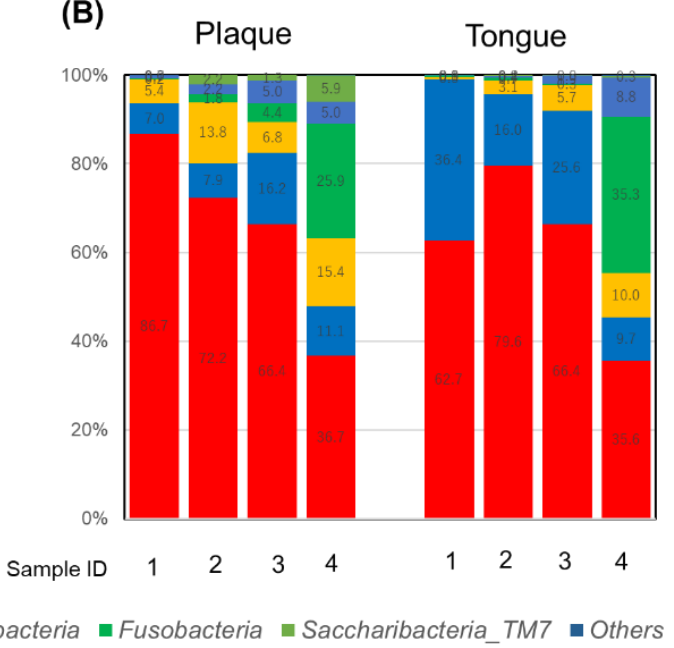

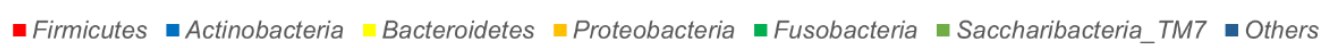

Figure 1. Proportion of phyla in denture plaque and the tongue: (A) total, and (B) individual subjects.

\subsection{Core Microbiome Analysis}

Figure 2 shows the prevalence of taxa in terms of abundance. Abundant species belonged to Firmicutes, Proteobacteria, and Bacteroides. In this study, 29 species were detected from all subjects in denture plaque and the tongue. Table 1 shows the species detected from all subjects, and Table S3 shows the 15 species in plaque and 7 species in the tongue. The most abundant bacteria were Firmicutes at the phylum level and the Streptococcus salivarius group at the species level in both plaque and the tongue. The Fusobacterium nucleatum group was detected from all subjects. A heatmap of the candidates for the core microbiome is shown in Figure S3. 
Table 1. Bacteria detected in denture plaque and tongue from all subjects.

\begin{tabular}{|c|c|c|c|c|}
\hline \multirow{2}{*}{ Phylum } & \multirow{2}{*}{ Genus } & \multirow{2}{*}{ Species } & \multicolumn{2}{|c|}{ Abundance (\%) } \\
\hline & & & Plaque & Tongue \\
\hline \multirow{18}{*}{ Firmicutes } & \multirow{8}{*}{ Streptococcus } & Streptococcus salivarius group & 9.72 & 25.06 \\
\hline & & Streptococcus sinensis group & 4.12 & 13.22 \\
\hline & & Streptococcus pneumoniae group & 4.46 & 1.92 \\
\hline & & Streptococcus parasanguinis group & 2.30 & 3.83 \\
\hline & & Streptococcus gordonii group & 4.80 & 0.62 \\
\hline & & Streptococcus peroris group & 0.82 & 1.03 \\
\hline & & Streptococcus sanguinis & 0.87 & 0.08 \\
\hline & & Streptococcus_uc & 0.40 & 0.32 \\
\hline & \multirow{4}{*}{ Veillonella } & Veillonella dispar & 5.63 & 4.89 \\
\hline & & Veillonella parvula group & 7.40 & 0.51 \\
\hline & & Veillonella atypica & 1.90 & 1.95 \\
\hline & & Veillonella_uc & 0.05 & 0.09 \\
\hline & Gemella & Gemella haemolysans group & 0.23 & 0.54 \\
\hline & Granulicatella & Granulicatella adiacens group & 0.43 & 1.67 \\
\hline & Lachnoanaerobaculum & Lachnoanaerobaculum orale group & 0.13 & 0.08 \\
\hline & Lactobacillus & Lactobacillus salivarius & 3.95 & 0.33 \\
\hline & Megasphaera & Megasphaera micronuciformis & 0.29 & 0.07 \\
\hline & Moryella & Stomatobaculum longum & 0.17 & 0.22 \\
\hline \multirow{7}{*}{ Actinobacteria } & \multirow{3}{*}{ Actinomyces } & KE952139_s & 0.71 & 2.39 \\
\hline & & CAGY_s & 0.11 & 0.30 \\
\hline & & $J R M V \_s$ & 0.02 & 0.37 \\
\hline & Atopobium & Atopobium parvulum & 0.76 & 0.27 \\
\hline & \multirow{3}{*}{ Rothia } & Rothia mucilaginosa & 0.65 & 17.17 \\
\hline & & Rothia dentocariosa & 4.34 & 0.52 \\
\hline & & Rothia_uc & 0.02 & 0.10 \\
\hline \multirow{3}{*}{ Bacteroidetes } & \multirow{3}{*}{ Prevotella } & Prevotella histicola & 3.95 & 0.54 \\
\hline & & Prevotella jejuni & 0.32 & 1.06 \\
\hline & & Prevotella salivae & 0.35 & 0.08 \\
\hline Fusobacteria & Fusobacterium & Fusobacterium nucleatum group & 0.37 & 0.04 \\
\hline
\end{tabular}

Plaque

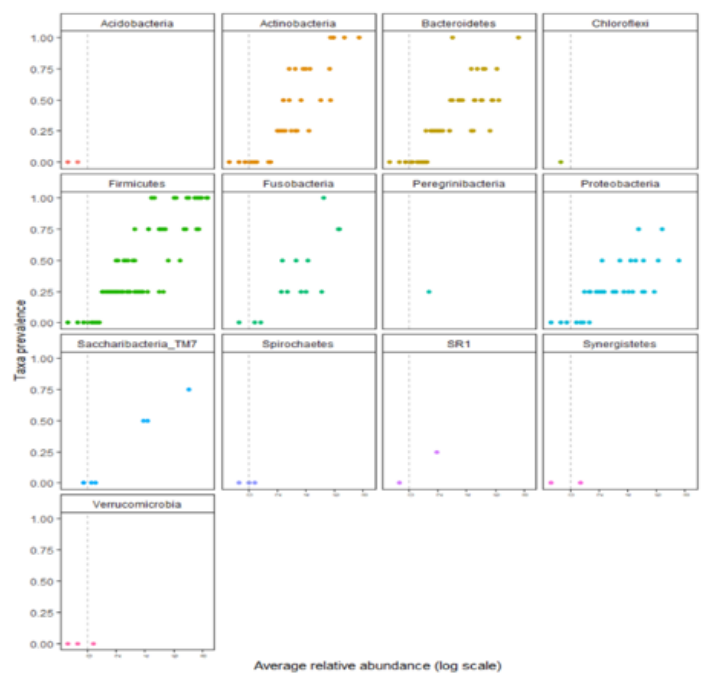

Tongue

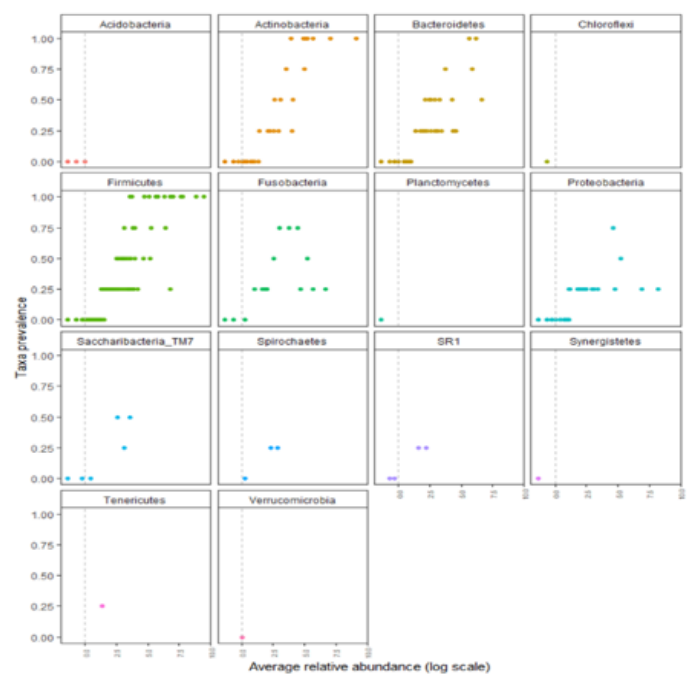

Figure 2. Prevalence of taxa in terms of abundance for 14 different phyla. 


\subsection{Analysis of Co-prevalent Species}

Then, the levels of species co-prevalence were analyzed by $\mathrm{t}$-distributed stochastic neighbor embedding (tSNE). As shown in Figure 3, species were constructed in clusters that were not dependent on the phylum. The list of classified species and detection in 3 of 4 subjects $(75 \%)$ is shown in Table S2.

(A)

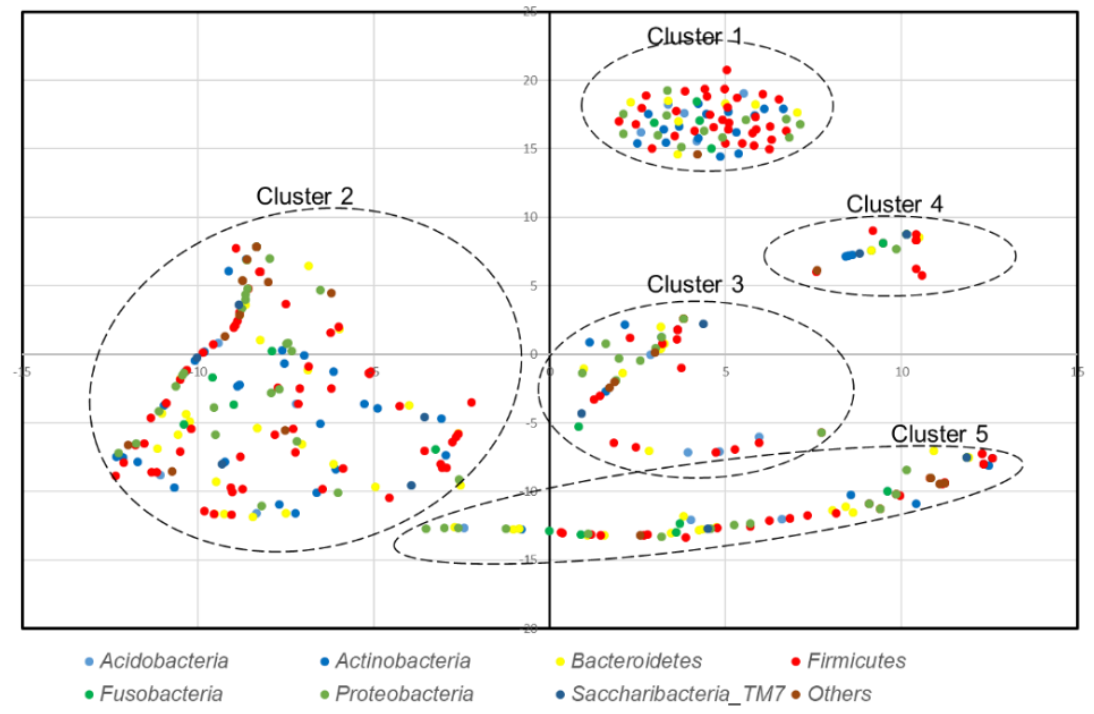

(B)

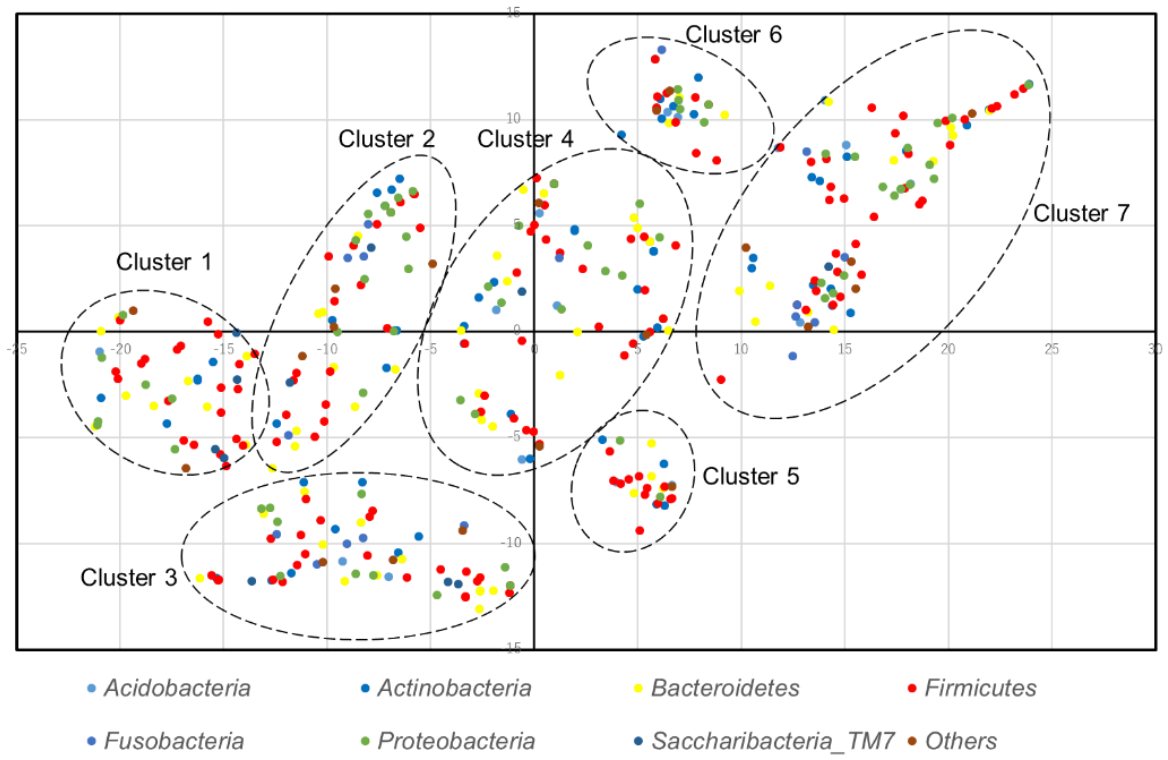

Figure 3. t-distributed stochastic neighbor embedding (tSNE) plot of species: (A) denture plaque, and $(\mathbf{B})$ the tongue.

\section{Discussion}

This study describes microbial profiles of the oral cavities of four female centenarians by high throughput sequencing. Highly prevalent and abundant species are described. In addition, differences in sampling sites and co-prevalence of species are presented.

A total of 406 species were detected. Studies indicate that the diversity of bacteria is reduced with the frailty of older adults $[3,46]$. All participants in this study were female centenarians. Bacterial diversity was not reduced when compared to another study [16]. 
Firmicutes was the most abundant phylum for both the denture plaque and tongue samples. Several studies have shown that Firmicutes is the most abundant phylum in the saliva of infants [16] and young children aged 6-8 years [47], swabs of the oral cavities of youths and adults [48], and dental plaque from older adults [34]. Firmicutes, Streptococcus, and Veillonella were detected in all subjects in both the denture plaque and the tongue. The result that Firmicutes was the most abundant, followed by Veillonella, is consistent with the proportions of oral microorganisms in the Human Oral Microbiome Database [49]. The abundant genera after Veillonella vary between studies. Streptococcus, Veillonella, and Neisseria were the predominant bacterial genera present in infants. Abundant genera that coat the tooth surface first are Streptococcus and Veillonella [50]. Streptococcus, Veillonella, and Neisseria are the predominant bacterial genera present in infants [16]. Streptococcus and Veillonella are known as early colonizers. Neisseria settles down at the age of 1-2 years [18].

In this study, Neisseria was not detected in all subjects. Differences at the species level in previous reports may be due to the sample difference. All subjects surveyed in this study wore complete dentures, so the plaque sample was denture plaque, not dental plaque attached to the tooth surface. Additionally, the small sample size may have affected the difference.

In this study, Streptococcus salivarius was the most abundant species in both the denture plaque and the tongue. This result is consistent with another study that investigated the tongues of Japanese older adults [16]. The results of the study show that more than $5 \%$ of abundant species were Streptococcus salivarius, Prevotella melaninogenica, Rothia mucilaginosa, Veillonella atypica, and Neisseria flavescens. Except for Neisseria flavescens, the other four species were common to our results. Another study showed that Streptococcus oralis was by far the most prevalent species [51]. As described above, Firmicutes and Veillonella are abundant phyla. Each study has different bacterial profiles at the genus or species level.

The bacterial profile of the denture plaque was similar to that of oral mucosa. Actinobacteria was abundant in the denture plaque and Bacilli were abundant in the dental plaque [52]. In this study, the proportion of these bacteria varies between samples: $5.4 \%, 15.5 \%, 10.8 \%$, and $6.5 \%$ for Actinobacteria and $82.45 \%, 44.4 \%, 21.5 \%$, and $48.1 \%$ for Bacilli. The small sample size may have affected the results.

A previous study had shown that oral microbiome profiles were related to the risk of death by pneumonia for older persons residing in nursing homes [53]. Neisseria flavescens, the Fusobacterium periodonticum group, and the Haemophilus parainfluenzae group were abundant in the risk group. In this study, one subject (Sample ID 4) had higher levels of these bacteria than the mean values of the risk group.

As shown in Figure 3 the bacterial community formed clusters with a low prevalence or abundance of bacteria. The clustering was not phylum dependent.

The clusters were composed of bacteria with a wide range of prevalence and abundance. This indicates that some bacteria were the core of the cluster. There may be an interaction or symbiotic mechanism between core bacteria and low-prevalence or low-abundance bacteria. Therefore, there may be healthy microbiome candidates within the core bacteria of each cluster.

A limitation of this study was the small sample size of four people. The design was a cross-sectional study. The data presented in this study were from Japanese older persons. Even though dietary habits have little effect on the oral microbiome [54], regional or cultural differences could have an effect on the oral microbiome [44]. The health status of the subjects who participated in this study was limited. Subjects with specific diseases were not included. For comparison, healthy and pathogenic conditions should be considered. Longevity is not simply explained by microflora. Further study is needed to evaluate the host factors such as nutraceutical agent [29], serum malondialdehyde [30], and progenitor cell levels [31]. In addition, the subjects investigated in this study were all female and edentulous.

\section{Conclusions}

The oral microbiome was preserved in Japanese centenarians at the phylum level. At the species level, bacterial profiles were not consistent with other studies. Firmicutes and Veillonella were abundant 
phyla in both the plaque and the tongue. In the bacterial profile, bacteria formed clusters. The oral microbiome of the centenarian investigated in this study was similar to that of other age groups from previous studies at phylum level. Further study is needed to define a common bacterial profile at the species level.

Supplementary Materials: The following are available online at http://www.mdpi.com/2076-3417/10/15/5312/s1. Figure S1: Heatmap of all species detected. Figure S2: Rarefaction curve. Figure S3: Core heatmap. Table S1: Alpha diversity indices of different groups. Table S2: Statistics of taxonomic assignment. Table S3: Species detected in denture plaque and tongue. Sequence data: Sequence data of the eight samples. Dynamic pie chart: Dynamic pie chart of the bacterial profiles of samples. OTU data: OTU data used in this study.

Author Contributions: Y.N. planned the study design and management and analysis of the data and wrote the original draft. E.K., A.O., R.O., M.S., Y.T., C.T., and K.A. collected and managed the data. Y.N., H.D., T.S., and N.H. contributed to funding acquisition, planning the study design, and reviewing and editing the manuscript. All authors have read and agreed to the published version of the manuscript.

Funding: This study was supported by JSPS KAKENHI (grant numbers 17K12030, 20K10303), SECOM Science and Technology Foundation, and an 8020 Research Grant for fiscal year 2017 from the 8020 Promotion Foundation (grant number 17-2-05). None of the funders played a role in the design of the study, data collection, analysis, interpretation of the results, or writing of the manuscript.

Conflicts of Interest: The authors state that they have no financial or nonfinancial conflict of interest regarding this research.

\section{References}

1. Yatsunenko, Y.; Rey, F.E.T.; Manary, M.J.; Trehan, I.; Dominguez-Bello, M.G.; Contreras, M.; Magris, M.; Hidalgo, G.; Baldassano, R.N.; Anokhin, A.P.; et al. Human gut microbiome viewed across age and geography. Nature 2012, 486, 222-227. [CrossRef]

2. Chung, H.Y.; Lee, E.K.; Choi, Y.J.; Kim, J.M.; Kim, D.H.; Zou, Y.; Kim, C.H.; Lee, J.; Kim, H.S.; Kim, N.D.; et al. Molecular inflammation as an underlying mechanism of the aging process and age-related diseases. J. Dent. Res. 2011, 90, 830-840. [CrossRef] [PubMed]

3. Claesson, M.J.; Cusack, S.; O'Sullivan, O.; Greene-Diniz, R.; de Weerd, H.; Flannery, E.; Marchesi, J.R.; Falush, D.; Dinan, T.; Fitzgerald, G.; et al. Composition, variability, and temporal stability of the intestinal microbiota of the elderly. Proc. Natl. Acad. Sci. USA 2011, 108, 4586-4591. [CrossRef]

4. O'Toole, P.W.; Jeffery, I.B. Gut microbiota and aging. Science 2015, 350, 1214-1215. [CrossRef]

5. Jackson, M.A.; Jeffery, I.B.; Beaumont, M.; Bell, J.T.; Clark, A.G.; Ley, R.E.; O’Toole, P.W.; Spector, T.D.; Steves, C.J. Signatures of early frailty in the gut microbiota. Genome Med. 2016, 8, 8. [CrossRef]

6. Zapata, H.J.; Quagliarello, V.J. The microbiota and microbiome in aging: Potential implications in health and age-related diseases. J. Am. Geriatr. Soc. 2015, 63, 776-781. [CrossRef]

7. Rampelli, S.; Candela, M.; Turroni, S.; Biagi, E.; Collino, S.; Franceschi, C.; O’Toole, P.W.; Brigidi, P. Functional metagenomic profiling of intestinal microbiome in extreme ageing. Aging 2013, 5, 902-912. [CrossRef]

8. Sampaio-Maia, B.; Monteiro-Silva, F. Acquisition and maturation of oral microbiome throughout childhood: An update. Dent. Res. J. 2014, 11, 291-301.

9. Flores, G.E.; Caporaso, J.G.; Henley, J.B.; Rideout, J.R.; Domogala, D.; Chase, J.; Leff, J.W.; Vázquez-Baeza, Y.; Gonzalez, A.; Knight, R.; et al. Temporal variability is a personalized feature of the human microbiome. Genome Biol. 2014, 15, 531. [CrossRef] [PubMed]

10. Teng, F.; Yang, F.; Huang, S.; Bo, C.; Xu, Z.Z.; Amir, A.; Knight, R.; Ling, J.; Xu, J. Prediction of early childhood caries via spatial-temporal variations of oral microbiota. Cell Host Microbe 2015, 18, 296-306. [CrossRef] [PubMed]

11. Boix-Amorós, A.; Collado, M.C.; Mira, A. Relationship between milk microbiota, bacterial load, macronutrients, and human cells during lactation. Front Microbiol. 2016, 7, 492. [CrossRef] [PubMed]

12. Fernández, L.; Langa, S.; Martín, V.; Maldonado, A.; Jiménez, E.; Martín, R.; Rodríguez, J.M. The human milk microbiota: Origin and potential roles in health and disease. Pharmacol. Res. 2013, 69, 1-10. [CrossRef] [PubMed] 
13. Fitzstevens, J.L.; Smith, K.C.; Hagadorn, J.I.; Caimano, M.J.; Matson, A.P.; Brownell, E.A. Systematic review of the human milk microbiota. Nutr. Clin. Pract. 2016, 32, 354-364. [CrossRef] [PubMed]

14. Rodriguez, J.M. The origin of human milk bacteria: Is there a bacterial entero-mammary pathway during late pregnancy and lactation? Adv. Nutr. Int. Rev. J. 2014, 5, 779-784. [CrossRef]

15. Gomez, A.; Nelson, K.E. The oral microbiome of children: Development, disease, and implications beyond oral health. Microb. Ecol. 2017, 73, 492-503. [CrossRef]

16. Cephas, K.D.; Kim, J.; Mathai, R.A.; Barry, K.A.; Dowd, S.E.; Meline, B.S.; Swanson, K.S. Comparative analysis of salivary bacterial microbiome diversity in edentulous infants and their mothers or primary care givers using pyrosequencing. PLOS ONE 2011, 6, e23503. [CrossRef]

17. Lif Holgerson, P.; Öhman, C.; Rönnlund, A.; Johansson, I. Maturation of oral microbiota in children with or without dental caries. PLoS ONE 2015, 10, e0128534. [CrossRef]

18. Dzidic, M.; Collado, M.C.; Abrahamsson, T.; Artacho, A.; Stensson, M.; Jenmalm, M.C.; Mira, A. Oral microbiome development during childhood: An ecological succession influenced by postnatal factors and associated with tooth decay. ISME J. 2018, 12, 2292-2306. [CrossRef]

19. Chen, W.P.; Chang, S.H.; Tang, C.Y.; Liou, M.L.; Tsai, S.J.; Lin, Y.L. Composition Analysis and Feature Selection of the Oral Microbiota Associated with Periodontal Disease. Biomed. Res. Int. 2018, 2018, 3130607. [CrossRef]

20. Minty, M.; Canceil, T.; Serino, M.; Burcelin, R.; Tercé, F.; Blasco-Baque, V. Oral microbiota-induced periodontitis: A new risk factor of metabolic diseases. Rev. Endocr. Metab. Disord. 2019, 20, 449-459. [CrossRef]

21. Graves, D.T.; Corrêa, J.D.; Silva, T.A. The Oral Microbiota Is Modified by Systemic Diseases. J. Dent Res. 2019, 98, 148-156. [CrossRef] [PubMed]

22. Jensen, E.; Allen, G.; Bednarz, J.; Couper, J.; Peña, A. Periodontal risk markers in children and adolescents with type 1 diabetes: A systematic review and meta-analysis. Diabetes Metab. Res. Rev. 2020, e3368. [CrossRef] [PubMed]

23. Wang, J.; Yang, X.; Zou, X.; Zhang, Y.; Wang, J.; Wang, Y. Relationship between periodontal disease and lung cancer: A systematic review and meta-analysis. J. Periodontal Res. 2020. [CrossRef]

24. Nadim, R.; Tang, J.; Dilmohamed, A.; Yuan, S.; Wu, C.; Bakre, A.T.; Partridge, M.; Ni, J.; Copeland, J.R.; Anstey, K.J.; et al. Influence of periodontal disease on risk of dementia: A systematic literature review and a meta-analysis. Eur. J. Epidemiol. 2020. [CrossRef] [PubMed]

25. Priyamvara, A.; Dey, A.K.; Bandyopadhyay, D.; Katikineni, V.; Zaghlol, R.; Basyal, B.; Barssoum, K.; Amarin, R.; Bhatt, D.L.; Lavie, C.J. Periodontal Inflammation and the Risk of Cardiovascular Disease. Curr. Atheroscler. Rep. 2020, 22, 28. [CrossRef] [PubMed]

26. Orlandi, M.; Graziani, F.; D'Aiuto, F. Periodontal therapy and cardiovascular risk. Periodontol 20002020. [CrossRef]

27. Jepsen, S.; Suvan, J.; Deschner, J. The association of periodontal diseases with metabolic syndrome and obesity. Periodontol 2000 2020. [CrossRef]

28. Mulhall, H.; Huck, O.; Amar, S. Porphyromonas gingivalis, a Long-Range Pathogen: Systemic Impact and Therapeutic Implications. Microorganisms 2020, 8, 869. [CrossRef]

29. Isola, G.; Polizzi, A.; Iorio-Siciliano, V.; Alibrandi, A.; Ramaglia, L.; Leonardi, R. Effectiveness of a nutraceutical agent in the non-surgical periodontal therapy: A randomized, controlled clinical trial. Clin. Oral Investig. 2020. [CrossRef]

30. Isola, G.; Polizzi, A.; Santonocito, S.; Alibrandi, A.; Ferlito, S. Expression of Salivary and Serum Malondialdehyde and Lipid Profile of Patients with Periodontitis and Coronary Heart Disease. Int. J. Mol. Sci. 2019, 20, 6061. [CrossRef]

31. Isola, G.; Giudice, A.L.; Polizzi, A.; Alibrandi, A.; Patini, R.; Ferlito, S. Periodontitis and Tooth Loss Have Negative Systemic Impact on Circulating Progenitor Cell Levels: A Clinical Study. Genes 2019, 10, 1022. [CrossRef]

32. Asakawa, M.; Takeshita, T.; Furuta, M.; Kageyama, S.; Takeuchi, K.; Hata, J.; Ninomiya, T.; Yamashita, Y. Tongue Microbiota and Oral Health Status in Community-Dwelling Elderly Adults. mSphere 2018, 3. [CrossRef] [PubMed] 
33. Feres, M.; Teles, F.; Teles, R.; Figueiredo, L.C.; Faveri, M. The subgingival periodontal microbiota of the aging mouth. Periodontol 2000 2016, 72, 30-53. [CrossRef] [PubMed]

34. Jiang, Q.; Liu, J.; Chen, L.; Gan, N.; Yang, D. The Oral Microbiome in the Elderly with Dental Caries and Health. Front. Cell Infect. Microbiol. 2019, 8, 442. [CrossRef] [PubMed]

35. Iwauchi, M.; Horigome, A.; Ishikawa, K.; Mikuni, A.; Nakano, M.; Xiao, J.Z.; Odamaki, T.; Hironaka, S. Relationship between oral and gut microbiota in elderly people. Immun. Inflamm. Dis. 2019, 7, 229-236. [CrossRef] [PubMed]

36. Ogawa, T.; Hirose, Y.; Honda-Ogawa, M.; Sugimoto, M.; Sasaki, S.; Kibi, M.; Kawabata, S.; Ikebe, K.; Maeda, Y. Composition of salivary microbiota in elderly subjects. Sci. Rep. 2018, 8, 414. [CrossRef] [PubMed]

37. Zakaria, M.N.; Furuta, M.; Takeshita, T.; Shibata, Y.; Sundari, R.; Eshima, N.; Ninomiya, T.; Yamashita, Y. Oral microbiome in community-dwelling elderly and its relation to oral and general health conditions. Oral Dis. 2017, 23, 973-982. [CrossRef]

38. Nomura, Y.; Kakuta, E.; Okada, A.; Otsuka, R.; Shimada, M.; Tomizawa, Y.; Taguchi, C.; Arikawa, K.; Daikoku, H.; Sato, T.; et al. Effects of self-assessed chewing ability, tooth loss and serum albumin on mortality in 80-year-old individuals: A 20-year follow-up study. BMC Oral Health. 2020, 20, 122. [CrossRef]

39. World Health Organization. Oral Health Surveys: Basic Methods, 5th ed.; World Health Organization: Geneva, Switzerland, 2013.

40. Okada, M.; Hayashi, F.; Nagasaka, N. PCR detection of 5 Putative periodontal pathogens in dental plaque samples from children 2 to 12 years of age. J. Clin. Periodontol. 2001, 28, 576-582. [CrossRef]

41. Okada, A.; Sogabe, K.; Takeuchi, H.; Okamoto, M.; Nomura, Y.; Hanada, N. Characterization of specimens obtained by different sampling methods for evaluation of periodontal bacteria. J. Oral Sci. 2017, 59, 491-498. [CrossRef]

42. Kim, O.S.; Cho, Y.J.; Lee, K.; Yoon, S.H.; Kim, M.; Na, H.; Park, S.C.; Jeon, Y.S.; Lee, J.H.; Yi, H.; et al. Introducing EzTaxon-e: A prokaryotic $16 \mathrm{~S}$ rRNA gene sequence database with phylotypes that represent uncultured species. Int. J. Syst. Evol. Microbiol. 2012, 62, 716-721. [CrossRef] [PubMed]

43. Yoon, S.H.; Ha, S.M.; Kwon, S.; Lim, J.; Kim, Y.; Seo, H.; Chun, J. Introducing EzBioCloud: A taxonomically united database of 16S rRNA and whole genome assemblies. Int. J. Syst. Evol. Microbiol. 2017, 67, 1613-1617. [CrossRef]

44. Nomura, Y.; Otsuka, R.; Hasegawa, R.; Hanada, N. Oral Microbiome of Children Living in an Isolated Area in Myanmar. Int. J. Environ. Res. Public Health 2020, 17, 4033. [CrossRef] [PubMed]

45. Lahti, L.; Shetty, S. Introduction to the Microbiome R Package, 2020. Available online: https://microbiome. github.io/tutorials/ (accessed on 31 July 2020).

46. Saraswati, S.; Sitaraman, R. Aging and the human gut microbiota-from correlation to causality. Front. Microbiol. 2014, 5, 764. [CrossRef] [PubMed]

47. Xu, Y.; Jia, Y.H.; Chen, L.; Huang, W.M.; Yang, D.Q. Metagenomic analysis of oral microbiome in young children aged 6-8 years living in a rural isolated Chinese province. Oral Dis. 2018, 24, 1115-1125. [CrossRef]

48. Burcham, Z.M.; Garneau, N.L.; Comstock, S.S.; Tucker, R.M.; Knight, R.; Metcalf, J.L. Patterns of Oral Microbiota Diversity in Adults and Children: A Crowdsourced Population Study. Sci. Rep. 2020, 10, 2133. [CrossRef]

49. Forsyth Institutes. eHOMED Expanded Human Oral Microbiome Database. Available online: http: //www.homd.org/ (accessed on 31 July 2020).

50. Kolenbrander, P.E.; Palmer, R.J.; Periasamy, S.; Jakubovics, N.S. Oral multispecies biofilm development and the key role of cell-cell distance. Nat. Rev. Microbiol. 2010, 8, 471-480. [CrossRef]

51. Aas, J.A.; Paster, B.J.; Stokes, L.N.; Olsen, I.; Dewhirst, F.E. Defining the normal bacterial flora of the oral cavity. J. Clin. Microbiol. 2005, 43, 5721-5732. [CrossRef]

52. O’Donnell, L.E.; Robertson, D.; Nile, C.J.; Cross, L.J.; Riggio, M.; Sherriff, A.; Bradshaw, D.; Lambert, M.; Malcolm, J.; Buijs, M.J.; et al. The Oral Microbiome of Denture wearers is influenced by levels of natural dentition. PLoS ONE 2015, 10, e0137717. [CrossRef] 
53. Kageyama, S.; Takeshita, T.; Furuta, M.; Tomioka, M.; Asakawa, M.; Suma, S.; Takeuchi, K.; Shibata, Y.; Iwasa, Y.; Yamashita, Y. Relationships of Variations in the Tongue Microbiota and Pneumonia Mortality in Nursing Home Residents. J. Gerontol. A Biol. Sci. Med. Sci. 2018, 73, 1097-1102. [CrossRef]

54. De Filippis, F.; Vannini, L.; LaStoria, A.; Laghi, L.; Piombino, P.; Stellato, G.; Serrazanetti, D.I.; Gozzi, G.; Turroni, S.; Ferrocino, I.; et al. The same microbiota and a potentially discriminant metabolome in the saliva of omnivore, ovo-lacto-vegetarian and Vegan individuals. PLoS ONE 2014, 9, e112373. [CrossRef] [PubMed]

(C) 2020 by the authors. Licensee MDPI, Basel, Switzerland. This article is an open access article distributed under the terms and conditions of the Creative Commons Attribution (CC BY) license (http://creativecommons.org/licenses/by/4.0/). 